EPJ Web of Conferences 71, 00054 (2014)

DOI: $10.1051 /$ epjconf / 20147100054

(C) Owned by the authors, published by EDP Sciences, 2014

\title{
Thermodynamics of SU(2) quantum Yang-Mills theory and CMB anomalies
}

\author{
Ralf Hofmann ${ }^{1,2, a}$ \\ ${ }^{1}$ Institut für Theoretische Physik \\ Universität Heidelberg \\ Philosophenweg 16 \\ 69120 Heidelberg, Germany \\ ${ }^{2}$ Institut für Photonenforschung und Synchrotronstrahlung \\ Karlsruher Institut für Technologie \\ Hermann-von-Helmholtz Platz 1 \\ Eggenstein-Leopoldshafen, Germany
}

\begin{abstract}
A brief review of effective SU(2) Yang-Mills thermodynamics in the deconfining phase is given, including the construction of the thermal ground-state estimate in terms of an inert, adjoint scalar field $\phi$, based on non-propagating (anti)selfdual field configurations of topological charge unity. We also discuss kinematic constraints on interacting propagating gauge fields implied by the according spatial coarse-graining, and we explain why the screening physics of an $\mathrm{SU}(2)$ photon is subject to an electricmagnetically dual interpretation. This argument relies on the fact that only (anti)calorons of scale parameter $\rho \sim|\phi|^{-1}$ contribute to the coarse-graining required for thermalground-state emergence at temperature $T$. Thus, use of the effective gauge coupling $e$ in the (anti)caloron action is justified, yielding the value $\hbar$ for the latter at almost all temperatures. As a consequence, the indeterministic transition of initial to final plane waves caused by an effective, pointlike vertex is fundamentally mediated in Euclidean time by a single (anti)caloron being part of the thermal ground state. Next, we elucidate how a low-frequency excess of line temperature in the Cosmic Microwave Background (CMB) determines the value of the critical temperature of the deconfining-preconfining phase transition of an $\mathrm{SU}(2)$ Yang-Mills theory postulated to describe photon propagation, and we describe how, starting at a redshift of about unity, SU(2) photons collectively work 3D temperature depressions into the CMB. Upon projection along a line of sight, a given depression influences the present CMB sky in a cosmologically local way, possibly explaining the large-angle anomalies confirmed recently by the Planck collaboration. Finally, six relativistic polarisations residing in the SU(2) vector modes roughly match the number of degrees of freedom in cosmic neutrinos (Planck) which would disqualify the latter as radiation. Indeed, if interpreted as single center-vortex loops in confining phases of SU(2) Yang-Mills theories neutrino mass $m_{v}$ solely arises by interactions with an environment. Cosmologically, the CMB represents this environment, and thus one would expect that $m_{v}=\xi T$ where $\xi=O(1)$. In this model cosmic neutrinos are a small darkmatter contribution, conserved only together with the CMB fluid, influencing Baryonic Acoustic Oscillations during CMB decoupling.
\end{abstract}

ae-mail: r.hofmann@thphys.uni-heidelberg.de

This is an Open Access article distributed under the terms of the Creative Commons Attribution License 2.0, which permits unrestricted use, distribution, and reproduction in any medium, provided the original work is properly cited. 


\section{Effective Yang-Mills thermodynamics and SU(2) смв}

To understand non-Abelian quantum gauge theory requires unconventional thinking because, except for asymptotically large momentum transfer [1,2], the fundamental Yang-Mills field $A_{\mu}$ selfinteracts strongly. In a high-temperature situation a pure SU(N) Yang-Mills theory was argued a long time ago to be inaccessible by perturbative techniques [3]: the so-called soft magnetic sector of the theory is screened too weakly for an infrared instability of the small-coupling expansion to be avoided. Namely, starting at order six in the perturbative series, kinetic and interaction terms are comparable, contradicting the perturbative paradigm that free particle propagation is modified by small corrections only. On the other hand, a numerical approach to Yang-Mills thermodynamics, which relies on a discretisation of Euclidean spactime on a 4D point lattice, suffers from available spatial lattice sizes being too small for capturing the long-range correlations of the soft magnetic sector exhaustively [4]. In contrast to the expectation based on a naive substitution of momentum transfer by temperature $T$ in the zero-temperature perturbative running of the gauge coupling high-temperature Yang-Mills thermodynamics exhibits strong nonperturbative effects [5] which, paradoxically (see also [6]), drive the behaviour of propagating gauge-field fluctuations in a rapid, power-like way to the Stefan-Boltzmann limit which, however, is subject to a larger number of polarisations [7]. Note that the extraction of the nonperturbative lattice $\beta$ function is problematic since the perfect lattice action at finite lattice spacing $a$ is prohibitively expensive to construct and operate [8].

In $[7,9]$ the deconfining-phase thermodynamics of SU(2) and SU(3) Yang-Mills theories was addressed nonperturbatively and analytically. The starting point is the construction of unique thermal ground-state estimates in terms of stable, (anti)selfdual, that is, nonpropagating gauge-field configurations of topological charge modulus unity: Harrington-Shepard (HS) (anti)calorons [10]. A spatial coarse graining over these energy- and pressure-free field configurations in a particular (singular) gauge, which is facilitated by the unique computation of the kernel of a linear differential operator in terms of an average over space and caloron scale parameter $\rho$ of the covariant two-point function of the fundamental (anti)caloron field-strength tensor $F_{\mu \nu}$, yields the equation of motion of an effective, inert, and adjoint scalar field $\phi$. For later use, it is important to point out that the $\rho$ integral is strongly dominated by the upper cutoff [11]. The potential of field $\phi, V(\phi)=\operatorname{tr} \Lambda^{6} / \phi^{2}$, which represents an additively unique entity, is parameterized by a mass scale $\Lambda$. The latter emerges by integration of the condition that an Euler-Lagrange type, second-order equation and a BPS type, first-order equation simultaneously need to be satisfied by field $\phi$ in the coarse-grained equivalent of singular gauge: the winding gauge. As a consequence, $\phi$ 's modulus is determined as $|\phi|=\sqrt{\frac{\Lambda^{3}}{2 \pi T}}$ in supernatural units $\hbar=c=k_{B}=1$.

Apart from potential $V(\phi)$ the complete, Euclidean effective action density for the coarse-grained, propagating gauge field $a_{\mu}$ includes a term $\operatorname{tr} \frac{1}{2} G_{\mu \nu} G_{\mu \nu}$, where $G_{\mu \nu} \equiv \partial_{\mu} a_{\nu}-\partial_{\nu} a_{\mu}-i e\left[a_{\mu}, a_{\nu}\right] \equiv G_{\mu \nu}^{a} t_{a}$ ( $e$ the effective gauge coupling) and a gauge-invariant kinetic term $\operatorname{tr}\left(D_{\mu} \phi\right)^{2}$ for field $\phi$. This form of the effective action is uniquely determined by the following constraints: (i) coarse-graining over interacting, topologically trivial fluctuations does not lead to a change of the form of the fundamental Yang-Mills action [12], (ii) the effective action needs to be gauge invariant, and (iii) field $\phi$ is inert and thus cannot participate in any momentum transfer. While condition (i) forbids the occurrence of higher dimensional operators solely constructed from gauge field $a_{\mu}$, condition (iii) assures that, apart from the kinetic term, operators, which mix fields $a_{\mu}$ and $\phi$, are excluded. From $\operatorname{tr}\left(D_{\mu} \phi\right)^{2}$ one reads off an adjoint-Higgs-mechanism induced quasiparticle mass term $m_{a}^{2}=-2 e^{2} \operatorname{tr}\left[\phi, t_{a}\right]\left[\phi, t_{a}\right]$ 
associated with direction $a$ of the Lie algebra. For SU(2) and in unitary gauge $\phi=2|\phi| t_{3}$, which is reached from winding gauge by an admissible albeit singular gauge transformation, one obtains: $m^{2} \equiv m_{1}^{2}=m_{2}^{2}=4 e^{2} \frac{\Lambda^{3}}{2 \pi T}$ and $m_{3}=0$. Instead of inferring the massiveness of two out of three gauge modes from the action density, one may envisage a Dyson series for the propagator of $a_{\mu}$ subject to an interaction monomial $m_{a}^{2}\left(a_{\mu}^{a}\right)^{2}=-2 e^{2} \operatorname{tr}\left[\phi, t_{a}\right]\left[\phi, t_{a}\right]\left(a_{\mu}^{a}\right)^{2}$. An immediate consequence of field $\phi$ being inert then is that the massive modes $a_{\mu}^{1,2}$ always propagate with $p^{2}=m^{2}$ ( $p$ being the respective four-momentum) because absorption or emission of a finite-momentum mode by $a_{\mu}^{1,2}$, which would facilitate a shift away from this thermal-quasiparticle mass-shell, necessarily transfers momentum to field $\phi$. This, however, is excluded by $\phi$ 's very definition.

Demanding that, on the level of noninteracting quasiparticles, the effective theory is thermodynamically consistent (Legendre transformations for thermodynamical quantities hold in the effective theory as they do in the fundamental theory) yields a first-order evolution equation for $e$ as a function of $T$. The respective solution exhibits attractor behaviour, characterised by a plateau $e=\sqrt{8} \pi$ and a logarithmically thin pole at the critical temperature $\lambda_{c} \equiv \frac{2 \pi T_{c}}{\Lambda}=13.87$ of the deconfining-preconfining phase transition where monopoles are screened to masslessness and thus start to condense [5]. Computing radiative corrections subject to this $T$ dependence of $e$ yields a tightly controlled loop expansion which is conjectured to terminate at a finite irreducible order [13]. The reason for increasing loop orders to imply hierarchically decreasing contributions to quantities such as pressure, energy density, and polarisation tensors is the explosion of the number of independent kinematic constraints with respect to the number of integration variables. Kinematic constraints, which are simple to formulate in physical, Coulomb-unitary gauge (total Cartan and off-Cartan gauge fixing, respectively), arise because the momentum transfer through an effective four-vertex is contrained to be $|\phi|^{2}$ in each Mandelstam variable $s, t$, and $u$ and because the massless mode's four momentum $p$ needs to satisfy $\left|p^{2}\right| \leq|\phi|^{2}$. Note that scattering, mediated by a four-vertex in the effective theory, is computed as a coherent average over the contributions from each channel [14].

Let us now review the role of coupling $e$ in the effective theory [11]. Working in units where $c=k_{B}=1$ but $\hbar$ is re-instated as an action, the (dimensionless) exponent

$$
-\frac{\int_{0}^{\beta} d \tau d^{3} x \mathcal{L}_{\mathrm{eff}}^{\prime}\left[a_{\mu}\right]}{\hbar}
$$

in the weight belonging to fluctuating fields in the effective partition function ${ }^{1}$ can, in unitary gauge, be re-cast as

$$
-\operatorname{tr} \int_{0}^{\beta} d \tau d^{3} x\left(\frac{1}{2}\left(\partial_{\mu} \tilde{a}_{v}-\partial_{v} \tilde{a}_{\mu}-i e \sqrt{\hbar}\left[\tilde{a}_{\mu}, \tilde{a}_{v}\right]\right)^{2}-e^{2} \hbar\left[\tilde{a}_{\mu}, \tilde{\phi}\right]^{2}\right), \quad\left(\beta \equiv \frac{1}{T}\right),
$$

if we define $\tilde{a}_{\mu} \equiv a_{\mu} / \sqrt{\hbar}$ and $\tilde{\phi} \equiv \phi / \sqrt{\hbar}$ and assume these fields not to depend on $\hbar$ [15]. Because of terms $\propto \hbar^{0}$ in (2) the unit of $\tilde{a}_{\mu}$ is length ${ }^{-1}$ which is also true of $\tilde{\phi}$. Thus coupling $e$ has units of $1 / \sqrt{\hbar}$, and we have

$$
e=\frac{\sqrt{8} \pi}{\sqrt{\hbar}}
$$

almost everywhere in the deconfining phase. Only (anti)calorons of scale parameter $\rho \sim|\phi|^{-1}$ contribute to the emergence of field $\phi$ and thus to the thermal ground state as a whole. In other words, only those (anti)calorons, which takes place just below the level of resolution in the effective theory,

\footnotetext{
${ }^{1}$ Since $\phi$ is inert the factor, whose exponent is the potential-part of the effective action, can be pulled out of the partition function formulated in terms of effective fields.
} 
actually support the description of deconfining Yang-Mills thermodynamics in terms of fields $\phi$ and $a_{\mu}$. Therefore, it is justified to use the effective coupling $e$ when computing the action of a single, relevant (anti)caloron as $S_{(\mathrm{A}) \mathrm{C}}=\frac{8 \pi^{2}}{e^{2}}$. But Eq. (3) then implies that $S_{(\mathrm{A}) \mathrm{C}}=\hbar$ which suggests that the indeterminism of plane-wave scattering by isolated effective vertices occurs because in and out states are interpolated in imaginary time by a fundamental, topologically nontrivial field configuration of a definite action.

In [7] it was assumed that the critical temperature $T_{c}$ for the deconfining-preconfining phase transition of a particular SU(2) Yang-Mills theory associated with thermal photon propagation coincides with the present baseline temperature $T_{0}=2.725 \mathrm{~K}$ of the Cosmic Microwave Background (CMB). This speculation is supported by the balloon borne NASA mission Arcade 2 [16] and earlier terrestial radio-frequency CMB radiance measurements, see references in [16]. Their results indicate a highly significant increase of $\mathrm{CMB}$ line temperature for decreasing frequencies below $3 \mathrm{GHz}$ which was interpreted in [17] as a mild redistribution of the CMB Rayleigh-Jeans spectrum due to an onset of photon Meissner massiveness and an accompanying evanescence of low frequency modes. For the $\mathrm{SU}(2)$ photon to acquire a small Meissner mass $\left(m_{\gamma} \sim 100 \mathrm{MHz}\right)$, as suggested by the data, one requires $T_{c}$ to be slightly below $T_{0}$. For all practical purposes, however, one can set $T_{c}=T_{0}$, and we have introduced the names $\mathrm{SU}(2)_{\mathrm{CMB}}$ for the physical Yang-Mills theory of this scale and $V^{ \pm}$for the vector modes of this model whose common mass vanishes with $T^{-1 / 2}$ with increasing temperature $T$.

This paper is organised as follows: The next section gives arguments on why the $\mathrm{SU}(2)_{\mathrm{CMB}}$ photon and the topological defects of $\mathrm{SU}(2)_{\mathrm{CMB}}$ (in the deconfining phase: unresolved, screened monopoles; in the preconfining phase: condensed massless monopoles) enjoy an electric-magnetically dual interpretation. Also, results on the longitudinal and transverse parts of the polarisation tensor of the massless mode are reviewed. In Sec. 4 the afore mentioned low-frequency anomaly of CMB line temperature is described in more detail. Sec. 5 exposes the major CMB anomalies at large angles as recently confirmed by the results of the Planck collaboration. A scenario on how $\mathrm{SU}(2)_{\mathrm{CMB}}$ can possibly explain these anomalies is discussed in Sec. 6. Finally, Sec. 7 is devoted to the apparent problem of $\mathrm{SU}(2)_{\mathrm{CMB}}$ yieding too many relativistic degrees of freedom appear at big-bang nucleosynthesis and CMB decoupling: Already at moderate redshifts the $\mathrm{SU}(2)_{\text {Смв }}$ vector modes $V^{ \pm}$nearly match the number of massless neutrino degrees of freedom as extracted from the Planck data. This leads to the proposition that the latter should be replaced by the former. Cosmic neutrinos, in turn, are argued to behave like (luke-)warm dark matter: Interpreted as single center-vortex loops in the confining phases of additional SU(2) Yang-Mills theories, their mass emerges by interaction with the CMB.

\section{Electric-magnetically dual interpretation in SU(2) $)_{\text {смв }}$}

If photon progation is, indeed, due to an $\mathrm{SU}(2)$ rather than a $\mathrm{U}(1)$ gauge principle then the observation that $e=\frac{\sqrt{8} \pi}{\sqrt{\hbar}}$ has implications. Namely, the question then arises how the unit of electric charge $Q$ is seen by the SU(2) Смв photon as compared to a conventional U(1) photon. In units of $c=k_{B}=1$ and with $\hbar$ re-instated as an action, this is addressed by recalling that the QED fine-structure constant $\alpha$, a unitless quantity, is given as

$$
\alpha=\frac{Q^{2}}{4 \pi \hbar} .
$$

For $\alpha$ to be unitless (in any system of units $\alpha$ is dimensionless) $Q$ should be proportional to $\sqrt{\hbar}$ which is the case if $Q \propto 1 / e$. Since this is true of magnetic charge w.r.t. to the Cartan subgroup U(1) of $\mathrm{SU}(2)$ (the charge of a monopole inside an (anti)caloron of extent $\rho \sim|\phi|^{-1}$ is $\frac{4 \pi}{e}$ ) we conclude that an electric field of the real world actually is a magnetic field in $\mathrm{U}(1) \in \mathrm{SU}(2)_{\mathrm{CMB}}$ and vice versa. That 


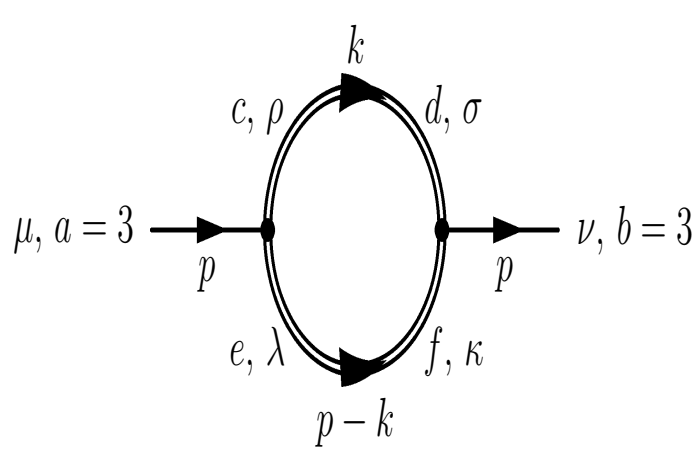

A

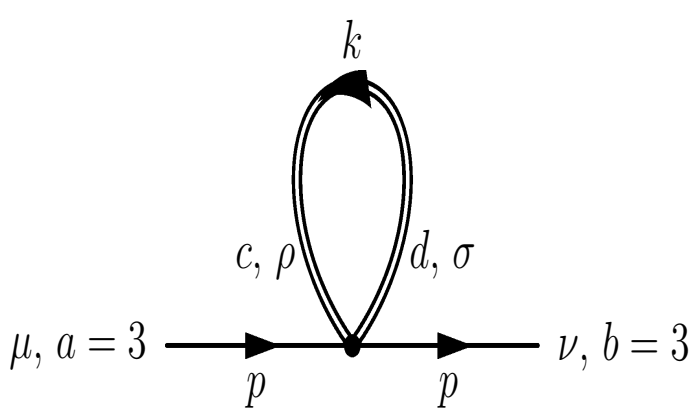

$\mathrm{B}$

Figure 1. The two diagrams potentially contributing to the one-loop polarisation tensor of $\gamma$. Double lines associate with $V^{ \pm}$propagation, a single line with $\gamma$.

is, an electric-magnetically dual interpretation of electromagnetism based on $\mathrm{SU}(2)_{\mathrm{CMB}}$ is in order ${ }^{2}$. Thus $\mathrm{SU}(2)_{\mathrm{CMB}}$ predicts the existence of electric rather than magnetic monopoles. These monopoles, however, are never resolved in the effective theory: they collectively contribute to the alteration of conventional, thermal photon propagation.

\section{Polarisation tensor of the thermal photon}

Here we review briefly how (anti)screening effects due to interaction with the vector modes $V^{ \pm}$influence the propagation properties of the photon $\gamma$ in $\mathrm{SU}(2)_{\text {смв }}$. There are two invariants $G\left(p_{0}, \vec{p}, T\right)$ and $F\left(p_{0}, \vec{p}, T\right)$ which modify the respective dispersion laws for tranverse and longitudinal ${ }^{3}$ photon propagation of four momentum $\left(p_{0}, \vec{p}\right)$ in a thermal plasma containing $\gamma$ and $V^{ \pm}$. Collectively, interaction of $\gamma$ with $V^{ \pm}$in the effective theory summarises screening and antiscreening effects the photon experiences due to the presence of unresolved electric monopoles induced into the thermal groundstate estimate by effective radiative corrections [5]. On a fundamental level, induction of monopole constituents is a consequence of transient holonomy shifts in (anti)calorons [19-22] due to their interaction with the propagating sector. Depending on the acquired holonomy, induced monopoleantimonopole pairs either attract one another (small holonomy), leading to annihilation, or, rarely, their repulsion (large holonomy) causes the (anti)caloron to dissociate into these constituents which then are subject to screening by other (anti)monopoles. We have argued and shown in [13,23, 24] that due to a large hierarchy in radiative corrections with increasing loop order it is sufficient for practical purposes to compute $\gamma$ 's polarisation tensor $\Pi_{\mu v}$ on the one-loop level.

In unitary-Coulomb gauge kinematic constraints allow only diagram B in Fig. 1 to contribute to $\Pi_{\mu \nu}$. For screening function $G$ the following gap equation arises [23, 25]:

\footnotetext{
${ }^{2}$ The Bianchi identities of the conventional theory are not violated since the vector modes $V^{ \pm}$of $\mathrm{SU}(2)_{\mathrm{CMB}}$, which are of magnetic charge in the real world, practically do not couple to the photon at electric field strengths prevailing in atoms and molecules and also for macroscopic electric field strengths, see discussion in [18].

${ }^{3}$ On tree level, there is no longitudinal polarisation, but magnetic charge density waves are generated radiatively in the Yang-Mills plasma.
} 

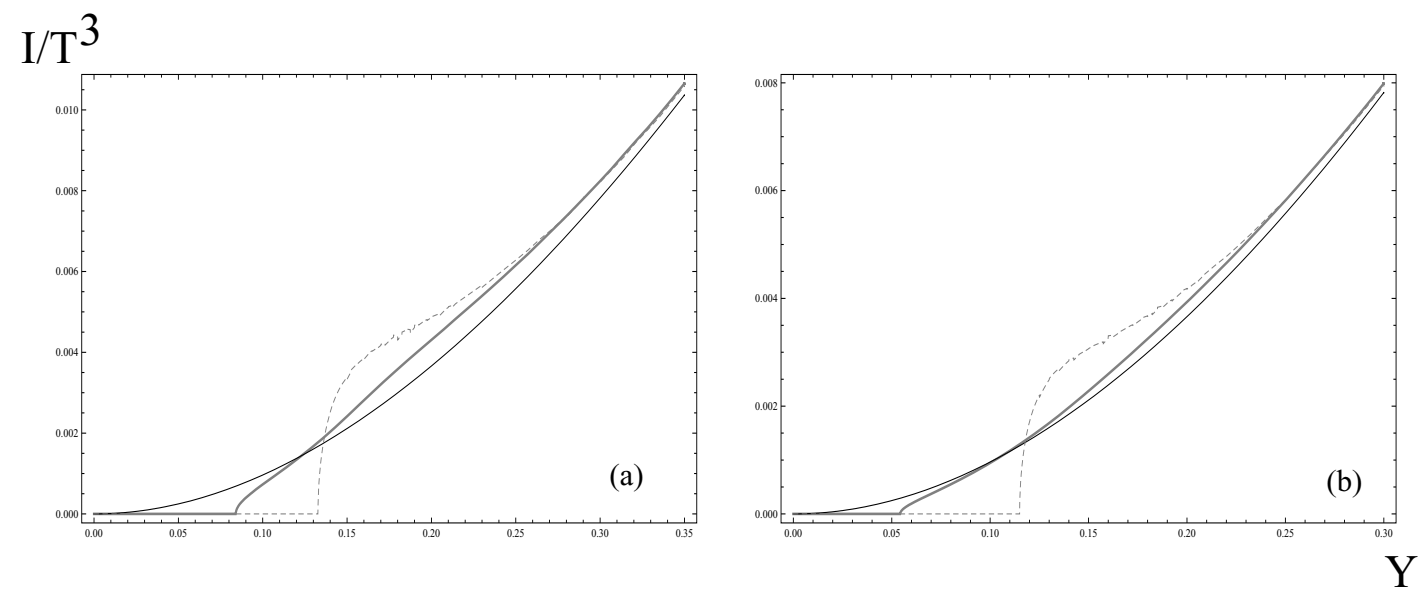

Figure 2. Plots of the results for the dimensionless spectral energy density $\frac{I}{T^{3}}$ obtained in the one-loop selfconsistent calculation (solid grey curves) and with the approximation $p^{2}=0$ (dashed grey curves) for (a) $T=3 T_{c}$ and (b) $T=4 T_{c}$. Here $Y \equiv \frac{p_{0}}{T}$. Solid black curves depict the conventional Rayleigh-Jeans spectrum. Note the much faster approach with rising temperature to the low-frequency Planck spectrum in the selfconsistent one-loop result in comparison with the approximate calculation.

$$
\begin{aligned}
\frac{G}{T^{2}} & =\frac{\Pi^{11}}{T^{2}}=\frac{\Pi^{22}}{T^{2}}= \\
& =\frac{e^{2}}{\pi \lambda^{3}} \int d^{3} y\left(-2+\frac{y_{1}^{2}}{4 e^{2}}\right) \frac{n_{B}\left(2 \pi \lambda^{-3 / 2} \sqrt{\vec{y}^{2}+4 e^{2}}\right)}{\sqrt{\vec{y}^{2}+4 e^{2}}},
\end{aligned}
$$

where the integration over dimensionless variables on the right-hand side is subject to the constraint

$$
\left|\frac{G}{T^{2}} \frac{\lambda^{3}}{(2 \pi)^{2}}+\frac{\lambda^{3 / 2}}{\pi}\left( \pm \sqrt{X^{2}+\frac{G}{T^{2}}} \sqrt{\rho^{2}+\xi^{2}+4 e^{2}}-X \xi\right)+4 e^{2}\right| \leq 1 .
$$

In (6) cylindrical coordinates $y_{1} \equiv \rho \cos \varphi, y_{2} \equiv \rho \sin \varphi$, and $y_{3}=\xi$ were introduced, and we define $X \equiv \frac{|\vec{p}|}{T}$. Similar expressions hold for $F$ 's gap equation [18]. The modification of transverse photon dispersion introduced by $G$ leads to an anomaly in the blackbody spectrum of energy density which is indicated in Fig. 2 [23, 25]. Longitudinal propagation occurs in various low-momentum branches. In [26] we have argued that these incoherent magnetic-field modes can acquire large correlation lengths if temperature $T$ exhibits an (adiabatically slow) dependence on space. In Sec. 6 this will be shown to happen on cosmological distances associated with a reshift of order unity by induction through the blackbody anomaly in transverse modes. So the longitudinal $\gamma$ sector could provide for emergent cosmic magnetic fields by virtue of CMB temperature inhomogeneities arising from anomalous thermal photon propagation.

\section{CMB low-frequency anomaly and SU(2) смв}

To make contact with $\mathrm{CMB}$ observation it is necessary to fix the Yang-Mills scale or, which is the same, $T_{c}$ of $\mathrm{SU}(2)_{\mathrm{CMB}}$ by observation. To this end, we discuss low-frequency observations of the CMB line temperature. 
Arcade 2 is a balloon borne instrument to observe the CMB at very low frequencies (bands at $v=3,8,10,30$, and $90 \mathrm{GHz}$ ) by alternating observation of a calibrator black body emitter and the CMB sky. After subtraction of known and well modelled foreground radiance in the sky signal, an adjustment of the calibrator's temperature such that the two signals null each other yields the temperature of the calibrator as a function of frequency (CMB line temperature). The interesting feature in this data was the detection of a clear excess (statistically significant at the level of five standard deviations) of the CMB line temperature $T(v)$ at 3 and $8 \mathrm{GHz}$. This is expressed by a powerlaw model [16]

$$
T(v)=T_{0}+T_{R}\left(\frac{v}{v_{0}}\right)^{\beta} .
$$

subject to the following parameter values: $T_{0}=2.725 \mathrm{~K}$ (within errors FIRAS' CMB baseline temperature [27]) obtained by a fit to the CMB spectrum at frequencies from $60 \mathrm{GHz}$ to $600 \mathrm{GHz}$ ), $v_{0}=310 \mathrm{MHz}, T_{R}=24.1 \pm 2.1 \mathrm{~K}$, and $\beta=-2.599 \pm 0.036$. The claim by the collaboration that this significant low-frequency deviation from a perfect black-body spectrum $(T(v) \equiv$ const) is not an artefact of galactic foreground subtraction, unlikely is related to an average effect of distant point sources, and that these results confirm the trend seen in earlier radio-frequency data [28-31] appears to rest on solid grounds. In [17] this observational situation was taken as motivation for an unconventional explanation of Eq. (7) which, at the same time, determines the value of $T_{c}$ for $\mathrm{SU}(2)_{\mathrm{CMB}}$.

Roughly speaking, the rise in line temperature can be explained if $\gamma$ modes become evanescent at low frequencies due to the onset of the Meissner effect (electric monopoles start to condense into a new ground state at $T_{0}$ ). This leads to a re-arrangement of spectral blackbody power at low frequencies: the power of evanescent modes is maximal at $v=0$ and matches that of propagating photons at a transition frequency which is comparable to the (effective and feeble ${ }^{4}$ ) photon mass of about $100 \mathrm{MHz}$. In $\mathrm{SU}(2)_{\mathrm{CMB}}$ this scenario takes place if $T_{c}$ is slightly below $T_{0}$, and, for all practical purposes, one may set $T_{c}=T_{0}$.

\section{CMB large-angle anomalies: WMAP and Planck}

Recently released data by the Planck collaboration confirm and strengthen the occurrence of largeangle anomalies in the CMB map of temperature fluctuations [32] which were first noticed based on WMAP data [33-36]. Here are some of them:

- Large-angle suppression of $T T(\theta)$, defined as the product of temperature in two pixels, which are separated by angle $\theta$, averaged over all possible pixel pairs. On angular scales $\theta>60^{\circ}$ and most notably on the northern ecliptic hemisphere (equatorial plane = plane of the solar system), $T T(\theta)$ is significantly smaller than predicted by the Cosmological Standard Model (CSM).

- Low variance of temperature fluctuations in the northern compared to the southern ecliptic hemisphere. The variance in the latter essentially agrees with CSM simulations.

- Power asymmetry. There is a plane, again close to the ecliptic, for which the difference between the average amplitude of temperature fluctuations in the northern and southern hemispheres reaches a maximum.

- The CMB 'cold spot' - a region of anomalously low temperature. The cold spot is detected by an estimation of the kurtosis of the distribution of temperature values in its surroundings, indicating a local violation of Gaussianity.

- Significant alignment of the CMB quadrupole with the octupole.

\footnotetext{
${ }^{4}$ In [17] it was assumed that monopole condensation at $T_{0}$ is not sufficiently strong to lead to an extra photon polarisation.
} 


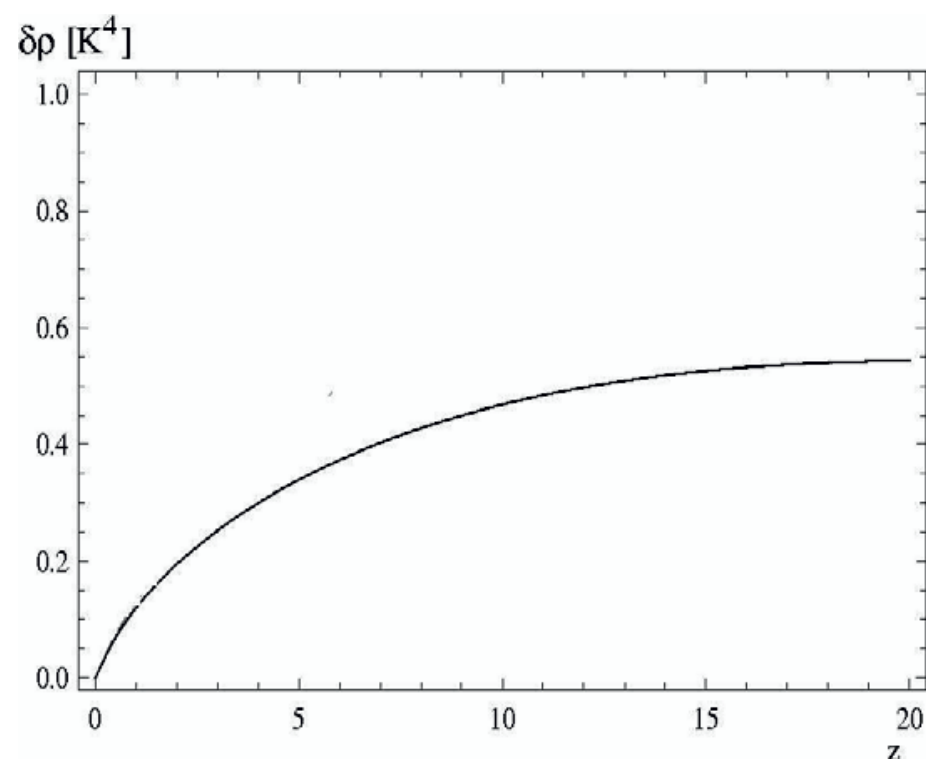

Figure 3. The difference $\delta \rho$ in energy densities of the $\gamma$ part in $\mathrm{SU}(2)_{\mathrm{CMB}}$ and of thermal photons in the conventional $\mathrm{U}(1)$ theory for photon propagation as a function of $z \equiv T / T_{0}-1$.

- Hemispherical antisymmetry. Temperature fluctuations in pixels that are related by mirror-reflection about a plane, whose normal is misaligned with the cold-spot direction by about $42^{\circ}$, tend to be the negative of one another.

A dipolar modulation model [37], which assumes a statistically isotropic CMB sky to be multiplicatively influenced by a vector $\vec{p}$, mimicks the actual CMB sky realistically [32].

\section{Dynamical breaking of the CMB's statistical isotropy by SU(2) смв $_{\text {s. }}$}

To discuss how $\mathrm{SU}(2)_{\mathrm{CMB}}$ could explain the $\mathrm{CMB}$ large-angle anomalies it is important to consider the difference $\delta \rho$ in energy densities, given by the respective spectral integrals, of the $\gamma$ part in $\mathrm{SU}(2)_{\mathrm{CMB}}$ and of thermal photons in the conventional U(1) theory for photon propagation, see Fig.2, as a function of $T$. Fig. 3 depicts $\delta \rho(T)$. Note that function $\delta \rho$ exhibits positive slope which is maximal at $T_{c}$. In propagating the CMB from decoupling at redshift $z \sim 1100$ to today $(z=0)$ its thermal photons should stream freely according to conventional theory modulo effects induced by nonlinear growth of matter structure (gravitational lensing, integrated Sachs-Wolfe) and re-ionised interstellar hydrogen/stellar plasmas (Sunyaev-Zeldovich). Disregarding these small effects, observed CMB temperature fluctuations $\delta T$ should be determined (i) by initial conditions, transferred from those of the matter sector through linear theory, and (ii) baryonic acoustic oscillations (BAO), influencing the CMB during decoupling. Note that angular fluctuations $\delta T$ are projections along lines of sights through $3 \mathrm{D}$ temperature distributions $\Delta T(z, \vec{x})$ for redshifts $z$.

If distribution $\Delta T$ is discretised on physical volume elements $\Delta V$, proportional to the third power of the FRW scale factor $a$, then a thermodynamical a priori probability density $P_{z}(\Delta T)$ for $\Delta T$ to occur at redshift $z$ within volume element $\Delta V$ can be assigned in the sense of a canonical ensemble (units 
are: $\left.k_{B}=\hbar=c=1\right)$

$$
P_{z}(\Delta T)=\frac{\exp \left(-\frac{\rho(\bar{T}+\Delta T) \Delta V}{\bar{T}}\right)}{\int_{T_{0}}^{\infty} d T \exp \left(-\frac{\rho(T) \Delta V}{\bar{T}}\right)}
$$

where now redshift $z$ relates to the average CMB temperature $\bar{T}$ as $z=\frac{\bar{T}}{T_{0}}-1$. Note that since the Silk cutoff for BAO translates today into a spatial scale equal to a typical galaxy diameter and because the energy density of today's CMB is $\rho_{\mathrm{U}(1)} \sim \frac{\pi^{2}}{15} T_{0}^{4}=\frac{\pi^{2}}{15}\left(11.9 \mathrm{~cm}^{-1}\right)^{4}$ the magnitude of the exponents in Eq. (8) is very large such that two very small numbers appear in the numerator and denominator defining $P_{z}(\Delta T)$. The factor $F_{z}$ to bias temperature fluctuations $\Delta T$ for $\mathrm{SU}(2)$ effects in cooling down the CMB by cosmological redshift should be the probability for $\Delta T$ to occur in volume element $\Delta V$ in $\mathrm{SU}(2)_{\mathrm{CMB}}$ normalized to the according $\mathrm{U}(1)$ probability ${ }^{5}$ :

$$
F_{z}(\Delta T)=\frac{P_{z, \mathrm{SU}(2)}(\Delta T)}{P_{z, \mathrm{U}(1)}(\Delta T)} \propto \exp \left(-\frac{\delta \rho(\bar{T}+\Delta T) \Delta V}{\bar{T}}\right) .
$$

Since $\delta \rho$ has positive slope, which vanishes, however, in a powerlike way for $\bar{T} \rightarrow \infty$ (see Fig. (3)), negative fluctuations $\Delta T$ are for $0 \leq z \leq 1$ significantly favoured over positive ones $\left(F_{z}(-|\Delta T|)>\right.$ $\left.F_{z}(|\Delta T|)\right)$ by prescribing

$$
\Delta T \rightarrow F_{z}(\Delta T) \Delta T
$$

If the propagation of the CMB to $z=0$ is performed in Fourier space then the multiplication of (10) turns into a convolution operation.

What are the implications? If by primordial chance $\Delta T_{\vec{x}}$ is negative within a given volume element $\Delta V_{\vec{x}}\left(\vec{x}\right.$ denoting a co-moving location at the center of $\Delta V_{\vec{x}}$ ) at a redshift $z \sim 1$ then the biasing of (10) strengthens this trend. That is, a local dip in temperature becomes deeper and, in going to lower redshift, broader due to the free-streaming part of the CMB photon spectrum cooling off the region contained in the forward light cone of $\vec{x}$. This leads to the emergence of cold regions of extent $z \sim 1$, their centers representing the locations of exceptionally negative, primordial seed fluctuations $\Delta T_{\vec{x}}$. Observation of the CMB from a present position at the slope of one of these cold regions or temperature depressions faces a preferred direction: the local gradient $\vec{p}$ of such a temperature depression. Statistical isotropy thus is broken locally by $\mathrm{SU}(2)_{\mathrm{CMB}}$ thermodynamics. It is clear that performing projections along and counter to the direction of $\vec{p}$ yields maximally asymmetric results: In the former case more unbiased fluctuations contribute, because the distance to the edge of the depression is small, and a variance asymmetry is expected as a consequence. Also, a dynamical contribution to the $\mathrm{CMB}$ dipole arises from the difference of these extremal projections while a cold spot emerges from their average. Because $\vec{p}$ emerges for $z<1$ it is clear that primordial fluctuations on large angular scales are most affected by the build-up of an observable temperature depression: this process suppresses the temperature-temperature correlation function for large angles, and it aligns low multipoles.

\footnotetext{
${ }^{5}$ In CMB simulations fluctuation $\Delta T$ is determined nonthermally in terms of Gaussian initial conditions, but $P_{z}(\Delta T) d T$ states how likely an apparent temperature parameter in a region represented by volume element $\Delta V$ would assume values in the range from $\bar{T}+\Delta T-\frac{1}{2} d T$ to $\bar{T}+\Delta T+\frac{1}{2} d T$ given an overall heat reservoir of physical, mean temperature $\bar{T}$. So long as there is no such physical reservoir, which is true of CMB photons after their last scattering if described by conventional U(1) gauge theory but also for $\mathrm{SU}(2)_{\mathrm{CMB}}$ photons if $\bar{T} \gg T_{0}, P_{z}(\Delta T)$ is an invalid concept. This is accomodated by the cancelation to unity between $\mathrm{SU}(2)_{\mathrm{CMB}}$ and $\mathrm{U}(1)$ in the definition of $F_{z}(\Delta T)$ in Eq. (9). However, for $\bar{T}$ larger but comparable to $T_{0}$ the thermal ground state of $\mathrm{SU}(2)_{\mathrm{CMB}}$ and the thermal ensemble of $V^{ \pm}$modes couple to $\gamma$ and thus commonly act as a heat reservoir. In the ratio $F_{z}(\Delta T)$ of $P_{z}(\Delta T)$ for $\mathrm{SU}(2)_{\mathrm{CMB}}$ and $\mathrm{U}(1)$ the $\mathrm{U}(1)$ part in $\mathrm{SU}(2)_{\mathrm{CMB}}$ still cancels. This part is due to non-Rayleigh-Jeans frequencies, which do not lend themselves $[18,25]$ to the concept of Eq. (8).
} 


\section{Cosmic neutrinos}

Let us finally address an interesting match of the non-photonic number of relativistic degrees of freedom extracted from the high- $l$ part of the angular power spectrum associated with the Planck data: [38] reports a number $N_{\text {eff }}$ of neutrino flavours as $N_{\text {eff }}=3.30 \pm 0.27$. Interestingly, this implies a number of about six relativistic degrees of freedom at $\mathrm{CMB}$ decoupling which is a near match to those represented by $V^{ \pm}$of $\mathrm{SU}(2)_{\text {смв }}$ : three relativistic polarisations in each of the two vector species. Note that already for $\bar{T}$ not much larger than $T_{0}=T_{c}$, the ratio $R$ of the (common) mass of $V^{ \pm}$to $\bar{T}$ is given as

$$
R=\frac{m_{V^{ \pm}}}{\bar{T}}=2 e \frac{|\phi|}{\bar{T}}=\sqrt{108} \pi^{2} \lambda_{c}^{-3 / 2}\left(\frac{\lambda}{\lambda_{c}}\right)^{-3 / 2} .
$$

For $\frac{\lambda}{\lambda_{c}}=2,10,1100$ one has $R=0.702,0.063,5.4 \times 10^{-5}$, respectively, and thus $V^{ \pm}$describe relativistic degrees of freedom already at moderate redshifts and certainly so during CMB decoupling. But if during CMB decoupling the vector modes $V^{ \pm}$of $\mathrm{SU}(2)_{\text {Смв }}$ actually play the role conventionally attributed to neutrinos then how do the latter vanish from the spectrum of relativistic degrees of freedom? In $[39,40]$ we have proposed that neutrinos could be single, that is, non-selfintersecting center-vortex loops $^{6}$ (zero selfintersection number, $N=0$ ) in the confining phases of pure SU(2) gauge theories whose Yang-Mills scales match the masses of charged leptons. Due to the absence of an explicit mass scale in the sector with $N=0$ and void of interactions with an environment a single vortex loop would shrink to a round point [40] of zero mass and vanishing electric/magnetic multipoles. However, cosmic neutrinos do interact with an environment, the $\mathrm{CMB}$, over long time scales. Already at moderate temperatures, the only CMB mass scale is $\bar{T}$, and thus we expect that neutrino masses are given as $m_{v}=\xi \bar{T}$ where $\xi$ is a dimensionless factor of order unity macroscopically parametrising the effect of the CMB on the mass of a center-vortex loop.

Is this compatible with neutrino oscillations physics? The strongest bound of $8 \times 10^{-3} \mathrm{eV}$ on neutrino mass scales comes from solar neutrino oscillations [41]. Since $T_{0}=2.35 \times 10^{-4} \mathrm{eV}$ this bound leads to the constraint $\xi \leq 30$. But already for values of $\xi$ moderately larger than unity neutrinos would behave like matter rather than radiation, see Fig. 4. Note that in this scenario CMB and neutrino fluids are no longer separately conserved. In CMB simulations $\xi$ should be included as a new fit parameter, and we expect a small, (luke-)warm contribution to dark matter to be mimicked by the neutrino sector during decoupling which should have an influence on BAO. Since the here-discussed scenario assumes that a thermal neutrino mass emerges by feeble interactions with the CMB the comparatively very local freeze-out physics at the onset of big-bang nucleosynthesis, where relative neutron and proton number densities are fixed by the equality of their conversion rate and the rate of expansion of the Universe, is not influenced: thermal masses of cosmic neutrino are induced after the fact, that is, only after their decoupling from matter.

\section{Summary and conclusion}

In this talk we have briefly reviewed the nonperturbative physics leading to the emergence of a thermal ground state in the deconfining phase of an SU(2) Yang-Mills theory, and we have discussed why the gauge symmetry breaking $\mathrm{SU}(2) \rightarrow \mathrm{U}(1)$, dynamically induced by this ground state - collectively representing calorons and anticalorons of topological charge modulus unity -, is commensurate with the postulate that photon propagation is fundamentally described by an $\mathrm{SU}(2)$ rather than a $\mathrm{U}(1)$

\footnotetext{
${ }^{6} \mathrm{~A}$ closed chain of nearly massless monopoles and antimonopoles, which, due to an electric-magnetically dual interpretation, carry feeble electric charges, locally move along and opposite to the tangential direction of the loop, and generate one unit of magnetic center flux.
} 


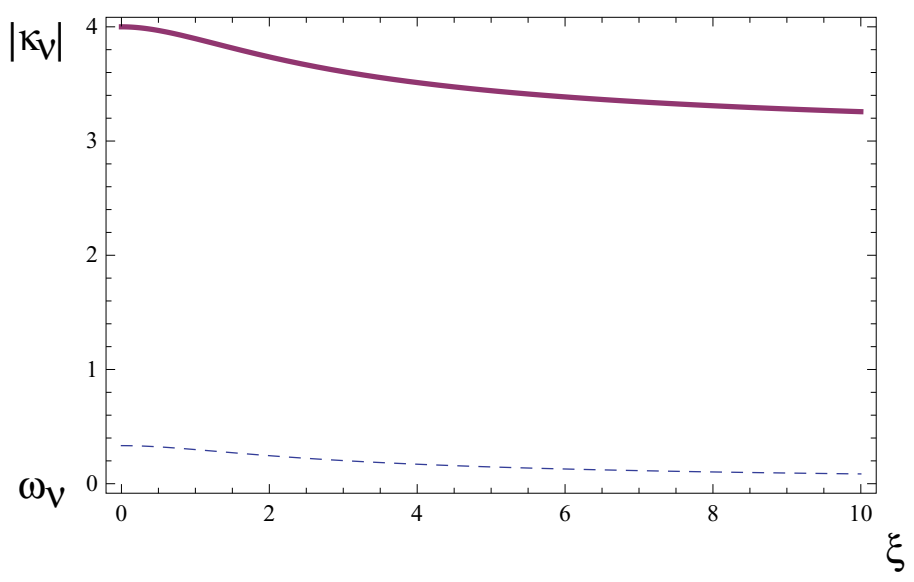

Figure 4. The modulus of $\kappa_{v}$ in $\rho(a)=\rho_{0} a^{k_{\nu}}$ (solid line), where $\rho, a$ denote energy density, scale factor, respectively $\left(a_{0} \equiv 1\right)$, and $\omega_{\nu}$ in $p=\omega_{\nu} \rho$ (dashed line), where $p$ denotes pressure, as functions of $\xi$ for cosmic neutrinos if the latter are assumed to be single center-vortex loops of pure $\mathrm{SU}(2)$ gauge theories in their confining phases. In this model, neutrinos would exhibit a CMB induced mass $m_{v}=\xi \bar{T}$.

gauge principle, see also [42]. In this context, explanations of a low-frequency anomaly in CMB line temperature [16] and recently confirmed [32] large-angle anomalies in the temperature fluctuations of the CMB sky are proposed which entail a re-interpretation of the number of relativistic degrees of freedom during CMB decoupling.

\section{Acknowledgments}

We would like to acknowledge useful conversations with James Annis and Markus Schwarz.

\section{References}

[1] D. J. Gross and F. Wilczek, Phys. Rev. Lett. 30, 1343 (1973).

[2] H. D. Politzer, Phys. Rev. Lett. 30, 1346 (1973).

[3] A. D. Linde, Phys. Lett. B 96, 289 (1980).

[4] J. Engels, F. Karsch, and T. Scheideler, Nucl. Phys. B 564, 302 (1999).

[5] J. Ludescher et al, Annalen. d. Physik 19, 102 (2010).

[6] H. M. Fried, Y. Gabellini, and T. Grandou, Annals Phys. 338, 107 (2013).

[7] R. Hofmann, Int. J. Mod. Phys. A 20, 4123 (2005); Erratum-ibid. A 21, 6515 (2006).

[8] P. Hasenfratz and F. Niedermayer, Nucl. Phys. B 414, 785 (1994).

[9] U. Herbst and R. Hofmann, ISRN High Energy Physics 2012, Article ID 373121 (2012).

[10] B. J. Harrington and H. K. Shepard, Phys. Rev. D 17, 2122 (1977).

[11] R. Hofmann, AIP Conf. Proc. 1479, 549 (2012).

[12] G. 't Hooft, G. and M. Veltman, Nucl. Phys. B 44, 189 (1972),

G. 't Hooft, G. and M. Veltman, Nucl. Phys. B 50, 318 (1972),

G. 't Hooft, G. and M. Veltman, Nucl. Phys. B 33, 173 (1971). 
[13] R. Hofmann, Braz. J. Phys. 42, 110 (2012).

[14] N. Krasowski and R. Hofmann, arXiv: 1301.4716 (2013).

[15] S. J. Brodsky and P. Hoyer, Phys. Rev. D 83, 045026 (2011).

[16] D. J. Fixsen et al, Astrophys. J. 734, 5 (2011).

[17] R. Hofmann, Ann. d. Phys. 18, 634 (2009).

[18] C. Falquez, R. Hofmann, and T. Baumbach, Ann. d. Phys. 522, 904 (2010).

[19] W. Nahm, in Monopoles in Quantum Field Teory, ed. N. Craigie et al. (World Scientific, Singapore), p. 87 (1982),

W. Nahm, in Trieste Group Theor. Method 1983, p. 189 (1983).

W. Nahm, CERN preprint TH-3172, (1981).

[20] K. Lee and C. Lu, Phys. Rev. D 58, 025011-1 (1998).

[21] T. C. Kraan and P. Van Baal, Phys. Lett. B 428, 268 (1998),

T. C. Kraan and P. Van Baal, Nucl. Phys. B 533, 627 (1998).

[22] D. Diakonov et al, Phys. Rev. D 70, 036003 (2004).

[23] M. Schwarz, R. Hofmann, and F. Giacosa, Int. J. Mod. Phys. A 22, 1213 (2007).

[24] D. Kaviani and R. Hofmann, Mod. Phys. Lett. A 22, 2343 (2007). on the one-loop level.

[25] J. Ludescher and R. Hofmann, Annalen d. Physik 18, 271 (2009).

[26] C. Falquez, R. Hofmann, and T. Baumbach, Quant. Matt. 1, 153 (2012).

[27] J. C. Mather et al, Astrophys. J. 420, 439 (1994).

[28] R. S. Roger et al, Astron. E Astrophys. Suppl. Ser. 137, 7 (1999).

[29] K. Maeda et al 140, 145 (1999).

[30] C. G. T. Haslam, Astron. E Astrophys. 100, 209 (1981).

[31] P. Reich and W. Reich, Astron. E Astrophys. Suppl. Ser. 63, 205 (1986).

[32] P. A. R. Ade et al, arXiv: 1305.5083.

[33] M. Tegmark, A. de Oliveira-Costa, and A. J. Hamilton, Phys. Rev. D 68, 123523 (2003).

[34] A. de Oliveira-Costa et al, Phys. Rev. D 69, 063516 (2004).

[35] C. J. Copi et al, Mon. Not. R. Astron. Soc. 367, 79 (2006).

[36] P. Vielva, Adv. Astron. 2010, Article ID 592094 (2010).

[37] C. Gordon, W. Huterer, and T. Crawford, Phys. Rev. D 72, 103002 (2005).

[38] P. A. R. Ade et al, arXiv: 1305.5076.

[39] F. Giacosa, and R. Hofmann, Eur. Phys. J. C 50, 635 (2007).

[40] J. Moosmann and R. Hofmann, ISRN Mathematical Physics 2012, Article ID 236783 (2012).

[41] M. Maltoni et al, New J. Phys. 6, 122 (2004).

[42] R. Hofmann, Nature Physics 9, 686 (2013). 